\title{
A Fusion Approach to IOT for Real Time Health Monitoring System
}

\author{
K Edison Prabhu ${ }^{\# 1}$, R Kannan ${ }^{* 2}$, \\ \# Department of EEE, Nehru Institute of Engineering and Technology, Coimbatore, Tamilnadu, India. \\ ${ }^{1}$ nietedisonprabhu@nehrucolleges.com \\ * Department of EEE, Nehru Institute of Engineering and Technology, Coimbatore, Tamilnadu, India. \\ 2rkannan_psg@yahoo.co.in
}

\begin{abstract}
Internet of Things (IOT) is the future technical approach to be going to make revolutionary changes in all the systematic fields "Future is in the hands of IOT". The wireless body area sensor network WBASN is one of the most advanced thinking in the area of e-medical field. WBASN improves the user experience by assuring outstanding quality in health care and patient monitoring system. In this paper introduce an advanced solution for IOT connected e-medical system by integrating IOT and WBASN, this model provides a possibility of monitoring the patient under $24 * 7$ period and analyze the health parameters of patient by health care professions anywhere, any time through their smart phone. Here the proposed model is developed by using various wireless body area sensors deployed on the different places of body for collect vital health parameters, then the collected data's are feed to an Arduino Galileo processor after that the preprocessed data's are upload to an open cloud server think Speak so that the health care professions check the real time health status of patient through their smart phone app thing Speak.
\end{abstract}

Keyword - IOT, WBASN, Arduino Galileo, Wi-Fi and Thing Speak

\section{INTRODUCTION}

The possibilities and popularity of internet provide new opportunities and ways, from this a new and ultimate paradigm is arises called Internet of Things IOT, IOT offers new ways of communication rather than computer to computer i.e., an ecosystem of inter connected peoples and devices and its success very soon gather attention from various and vast research fields and real time applications such as smart home, smart grid ,industrial internet, smart city, connected car, connected health and so on. In this work promote the health applications of IOT.

For a quality healthcare management and effective patient monitoring system, early detection of medical issues and emergency notifications are necessary, this is make possible with the help of an integrated system of wireless body area sensor network (WBSAN) and IOT, it provides a facility of automated, continues, unobtrusive monitoring of human physiological signals, this effectively collect the useful and health related information's from human body and access it. The necessary health parameters to be monitored are pulse rate, respiratory rate, body temperature, position of the body, blood glucose, ECG and so on. The next is connectivity method, the success of IOT based health system mainly depends on communication methods which promote the connection between WBASN and IOT. Here an Intel Galileo board is used for preprocess the sensor data's and Wi-Fi communication module is used for transmit the preprocessed data to cloud server, so the real time information's are upload to the cloud in a continues and automatic manner, here an open cloud server thing Speak is proposed, from the cloud the registered health practitioners are access the patient data's through their smart phone android app thing speak.

The following of the paper arranged as, next section 2 describes the works related with proposed model, and proposed design is explained in the section 3.The sections 4 and 5 respectively show the results and conclusions.

\section{RELATED WORKS AND MOTIVATION}

The popularity and possibilities of IOT dramatically improves in vast research fields and it is expected that in the year 2020 there will be more than 50 billion inter connected devices in the world and among this more than 8 billion are body area network devices (BAN). When recall the works related with IOT connected health system, there is some models existed for this purpose.

While considering the WBASN, different types of sensors are used. The AD18B20, LM 35 and MAX 30205 are some of the commonly used temperature sensors. The LM35 is a simple, low cost sensor but it's uncomfortable size and less sensitivity makes it unfit for many practical applications, the AD18B20 is a size compatible sensor and it have higher accuracy and sensitivity but its power consumption is slightly high, apart from LM 35 and AD18B20 the MAX 30205is comfortable in cost and have high rate of accuracy and sensitivity and it shows an ultra-low power consumption property so it is more suitable for real time applications. Next is body position analysis, there are two types of accelerometers generally used for identify the position of body, ADXL 335 and ADXL 362. The ADXL 335 is a cheap and fast sensing device when compared to ADXL 362, it 
is a very low power consuming device but its cost is more. For measure the pulse rate of patient photoplethysmography (PPG) sensor is more preferable, the other one available for this application is photo coupler (an arrangement of IR LED and photo diode) but its high power consumption and less sensitivity affects its suitability. The AD 8232 is an advanced ECG as well as EMG sensor used in BAN.

For preprocess the sensor data's a microcontroller or microprocessor unit is need, due to simplicity and user friendly characteristics the IDE boards are commonly preferred for this purpose. There are 3 types of IDE modules are commonly used Arduino UNO, Arduino Fio and Intel Galileo. The Arduino UNO is a simple, cheap, 8-bit micro controller unit and it have wide range of applications but its low clock speed and less flash memory limited its accessibility, apart from Arduino UNO the Arduino Fio is specially manufactured for interfacing the sensor applications. The third one Intel Galileo is an advanced microcontroller unit when compete with other Arduino modules, it has a clock speed of $400 \mathrm{MHz}$ and $8 \mathrm{Mb}$ flash memory, so it is the most suitable one for IOT applications.

There are several wireless communication modules are available for connect the WBASN to IOT, Wi-Fi, NFC, ZigBee, Bluetooth and UWB are some of the commonly preferred one's.

\section{PROPOSED MODEL}

The architecture of proposed IOT connected e-health system is illustrated in the above figure 1

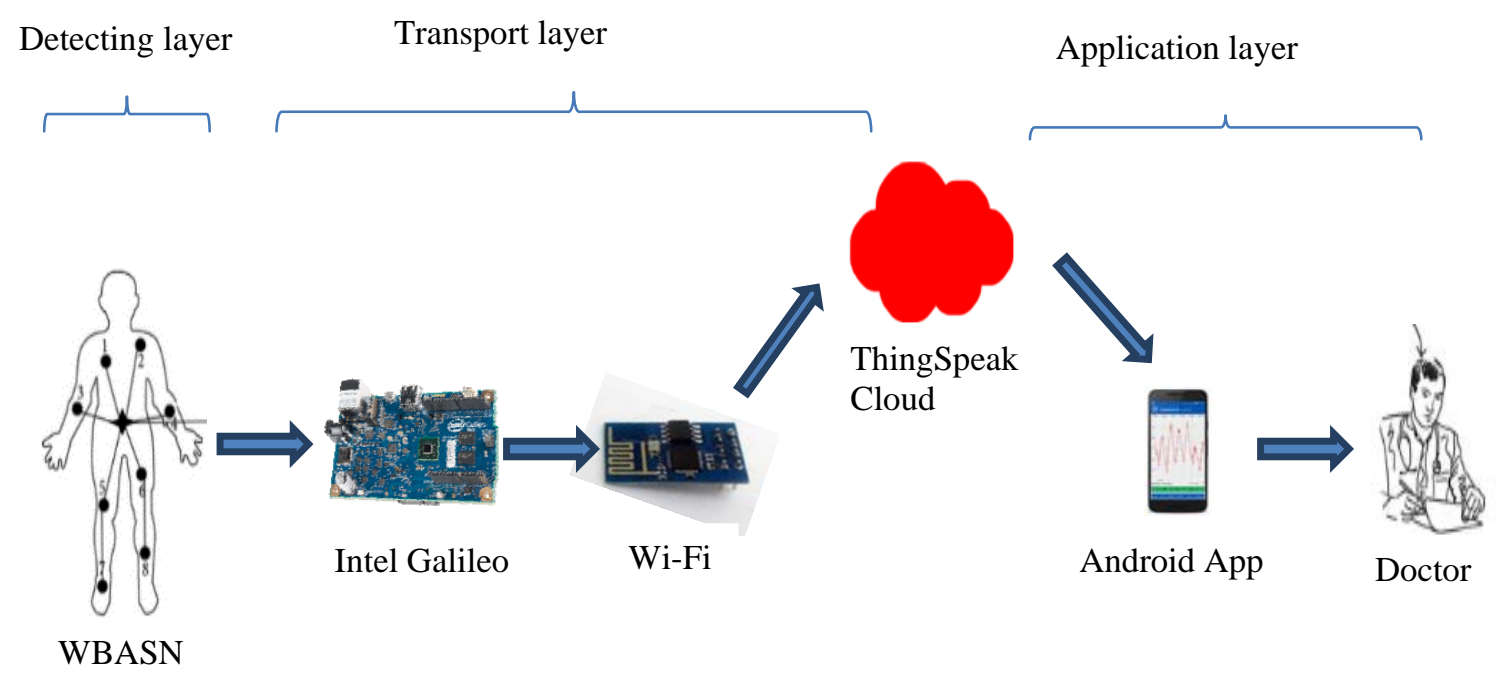

Figure 1: Architecture of proposed model.

In general, an IOT system can be classified into 3 layers, detecting layer, transport layer and application layer, the objective of detecting layer is the collection of real time data's here it is done by WBASN. Next the operation of transport layer is the transmission of collected information to a central server, the Intel Galileo board and Wi-Fi module is used for this purpose, the recovery of collected information from the central server is done in the application layer. In this model the open cloud server thing Speak is act as a central server and the android app thing Speak and its operating smart phone are belonging to application layer.

\section{A. WBASN}

Wearable sensors are the key components of wireless body area network(WABN), they collect the vital data of the subject for further usage and hence the medical professionals can able to routinely monitor the health status of subject. The following are such sensors chosen here.

\section{i. Accelerometer}

The ADXL 362 is one of the most suitable position analysis sensor manufactured by Analog Devices ${ }^{\mathrm{R}}$, its features make it adequate for this application such as 3-axis ultra-low power consuming MEMS device, having adjustable threshold limits and it provides a facility of "motion triggered wake-up mode" this feature helps to reduce the power consumption effectively because under fall detected conditions only it wake-up the processor and sent emergency notifications. The current consumptions under normal and wake-up modes are less than 2 $\mu \mathrm{A}$ and $170 \mu \mathrm{A}$ respectively. 


\section{ii. Temperature Sensor}

Because of high accuracy, resolution and reduced power consumption, the MAX 30205 from Maxim ${ }^{\mathrm{R}}$ is selected. By using this sensor, it is possible to evaluate the body temperature of subject at a rate of $0.1^{\circ} \mathrm{C}$ from $37^{\circ} \mathrm{C}$ to $39^{\circ} \mathrm{C}$, here the resolution is 16 bit, operating voltage is $2.7 \mathrm{~V}$ to $3.3 \mathrm{~V}$ and consumption of current is maximum up to $600 \mu \mathrm{A}$. Through an $\mathrm{I}^{2} \mathrm{C}$-compatible 2-wire serial interface method, this sensor is communicating with the MCU.

\section{iii. Pulse Sensor}

For monitor the heart pulses of subject, AD8232 module is chosen. It is a ECG as well as EMG sensor, it has the following specifications 2 or 3 electrode configuration, fully integrated single lead ECG front end, 170 $\mu \mathrm{A}$ typical current supply and operating voltage ranges from $2.0 \mathrm{~V}$ to $3.5 \mathrm{~V}$, the ultra-low power consuming analog to digital converter (ADC) and shutdown pin are its additional features. The sensing leads are deployed on the proper position of the subject's body like chest or wrist.

\section{B. INTEL GALILEO}

All the sensors deployed on the subject's body is interface with a microprocessor or micro controller unit for further analyze the collected data. Here the Intel Galileo a programmable control PCB, act as a processor is installed for this purpose. Why here it is chosen means, for a real time and long term application it shows a better performance and it has industrial standard $\mathrm{I} / \mathrm{O}$ ports and features and when compared to other equivalent modules like Intel core or Intel atom processors, it provides more cost effective development environment. In this Intel Galileo module, the used processor is Intel ${ }^{\mathrm{R}}$ Quark ${ }^{\mathrm{TM}} \mathrm{ScX} 100$ and its more specificati0on and ratings are mentioned in the following table 1.

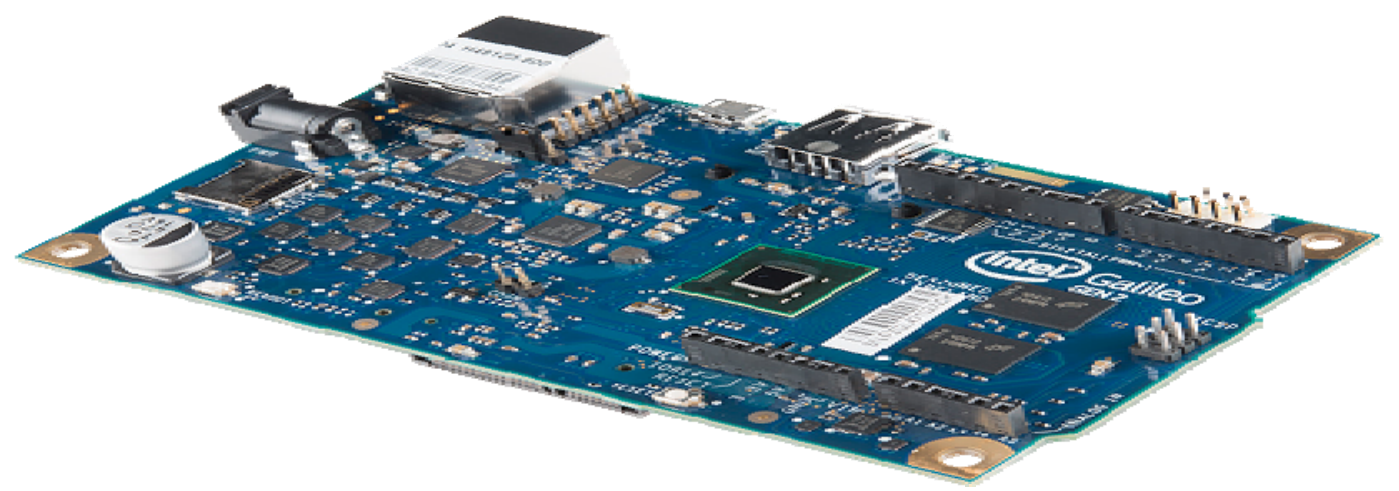

Figure 2: Intel Galileo Board

Table 1: Specification and Ratings of Arduino Galileo board

\begin{tabular}{|c|c|}
\hline Galileo Specification & Ratings \\
\hline Board dimensions & $10 \mathrm{~cm}$ x 7cm (slight overlap for power jack) \\
\hline Processor & Intel ${ }^{\mathrm{R}}$ Quark x 1000 - single core \\
\hline Description of processor & $\begin{array}{l}\text { Quark described by Intel at IDF2013, is very low power consumption, small } \\
\text { form factor, and low cost; ideal for "wearables," and the Internet of Things. }\end{array}$ \\
\hline Architecture & Intel ${ }^{\mathrm{R}}$ Pentinum ${ }^{\mathrm{R}}$ Class \\
\hline Speed & 400MHZ \\
\hline Width, Instruction Set & 32-bit \\
\hline Real Time Clock & Yes, needs a 3.3v coin cell \\
\hline Cache & 16KB L1 Cache \\
\hline RAM & $\begin{array}{l}\text { 512KB on-chip SRAM, dedicated for sketch storage \& 256MB DRAM, } \\
\text { dedicated for sketch storage }\end{array}$ \\
\hline FLASH Memory & 8MB NOR Flash (Legacy SPI), for FW bootloader \& Sketch storage \\
\hline EEPROM & $11 \mathrm{~KB}$ \\
\hline GPU & No \\
\hline External Storage & Micro-SD Card(up to 32GB),\& support for an external USB 2.0 drive \\
\hline
\end{tabular}




\section{WI-FI MODULE}

ESP 8266, a low cost Wi-Fi microchip made by Expressive Systems. In this work it is adopted for transmit the processed sensor data from processor unit to the open cloud server Thing speak. The ESP 8266module consists a 32-bit RISC microprocessor-L106, a 10 bit analog to digital converter and 16 GPIO pins. The supply voltage to the board is 3.3 and it has a clock speed of $160 \mathrm{MHz}$

\section{THING SPEAK}

For IOT applications a cloud server is necessary, the Thing Speak is a such one. It is an open source cloud server, supports the sensor logging applications, location tracking operations and helps to create a social network of things with status updates. In this proposed work it is selected for upload the subject's health data's and hence the registered medical professionals can able to monitor the real time health information's and view the current location of subject. Here a API key is required for store and retrieve the data from things and the used internet protocol is HTTP.

\section{EXPERIMENTAL RESULTS AND DISCUSSIONS.}

The functional arrangement of proposed model is illustrated in the figure 6 . It is seen that, the sensor nodes such as MAX 30205(body temperature), AD 8232(ECG and EMG) and ADXL 362(accelerometer) are deployed on the appropriate positions of subject's body and the sensors are inter facing with the Intel Galileo board to form WBASN. Next, the communication between Intel processor and Thing Speak cloud for transfer the data from processor to cloud is enabled by using a Wi-Fi module ESP 8266, the Wi-Fi module transmit the data to thing speak through a local Wi-Fi network. By using an android app Thing Speak and its operating smart phone, the data's can be retrieved.

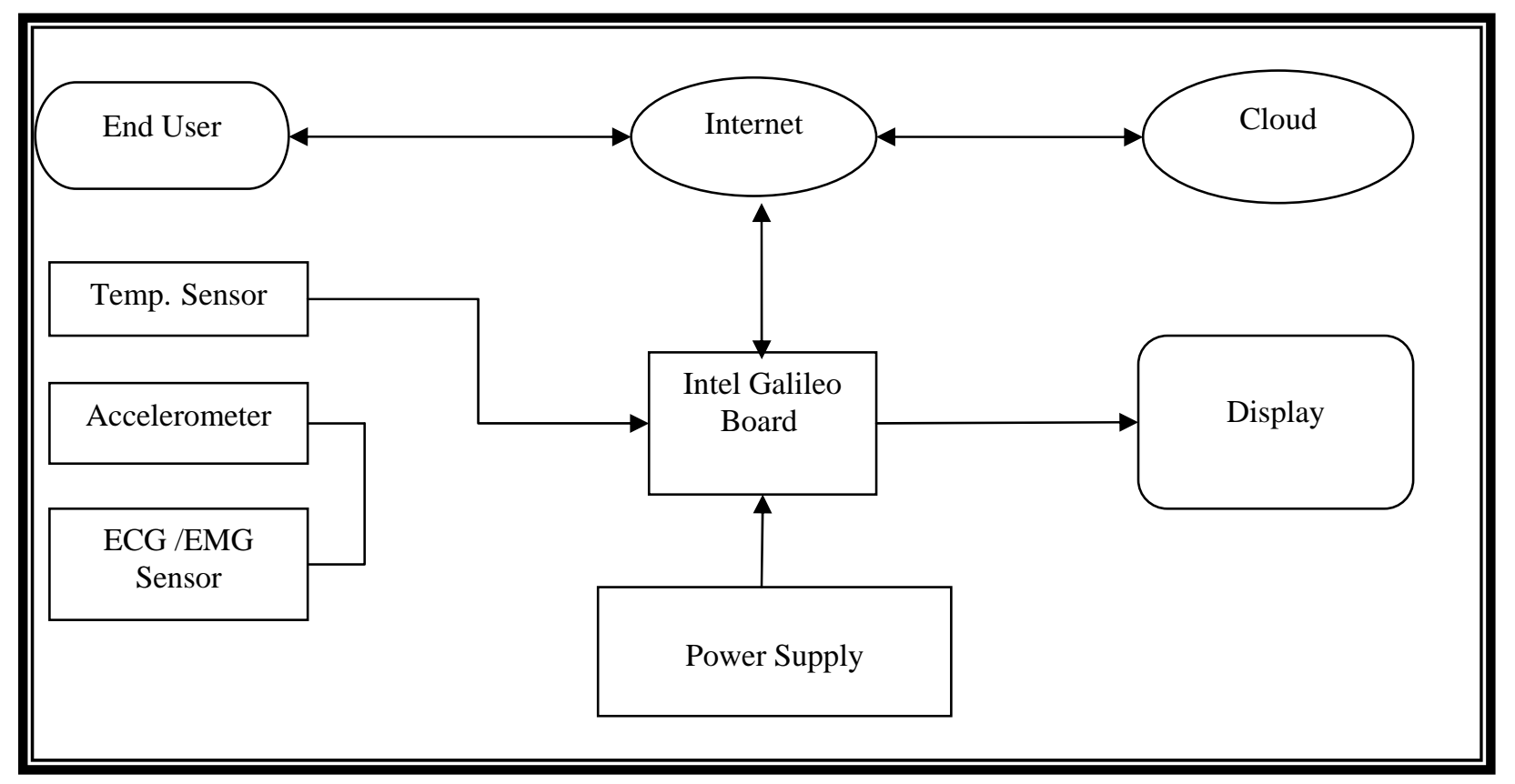

Figure 3: Functional view of proposed model.

1. Detecting layer

The collection of vital information's from subject by using appropriate sensors is the purpose of this detecting layer. Here the MAX 30502, a highly accurate temperature sensor has a sensing range from $37^{\circ} \mathrm{C}$ to $39^{\circ} \mathrm{C}$ is specified for human body temperature measurement, a ADXL 362 highly sensitive accelerometer and AD 8232, a ECG as well as EMG sensor are used for develop the detecting layer. These sensors are interface with the Intel processor and the sensor nodes are attached with the subject's body. 


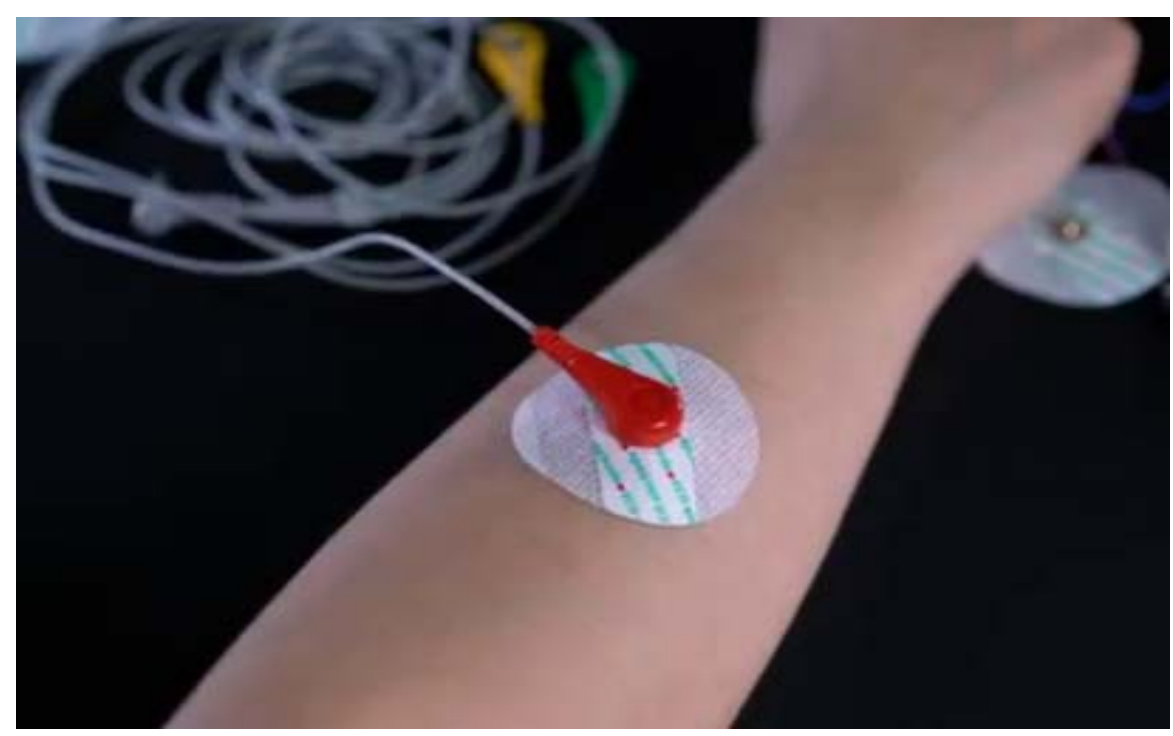

\section{Transport layer}

Figure 4: ECG/EMG sensor deployed on subject’s body

To enable the communication between detecting layer and application layers of IOT, transport layer is used. The wireless communication modules are belonging to this layer, in this work the ESP 8266, a microchip WiFi module is used in between the Intel processor and open cloud server ThingSpeak, as a data transport channel. For upload the data's to ThingSpeak cloud, it is necessary to create a ThingSpeak channel first then get a API key, this API key used in the application layer for recover the created channel and uploaded information's.

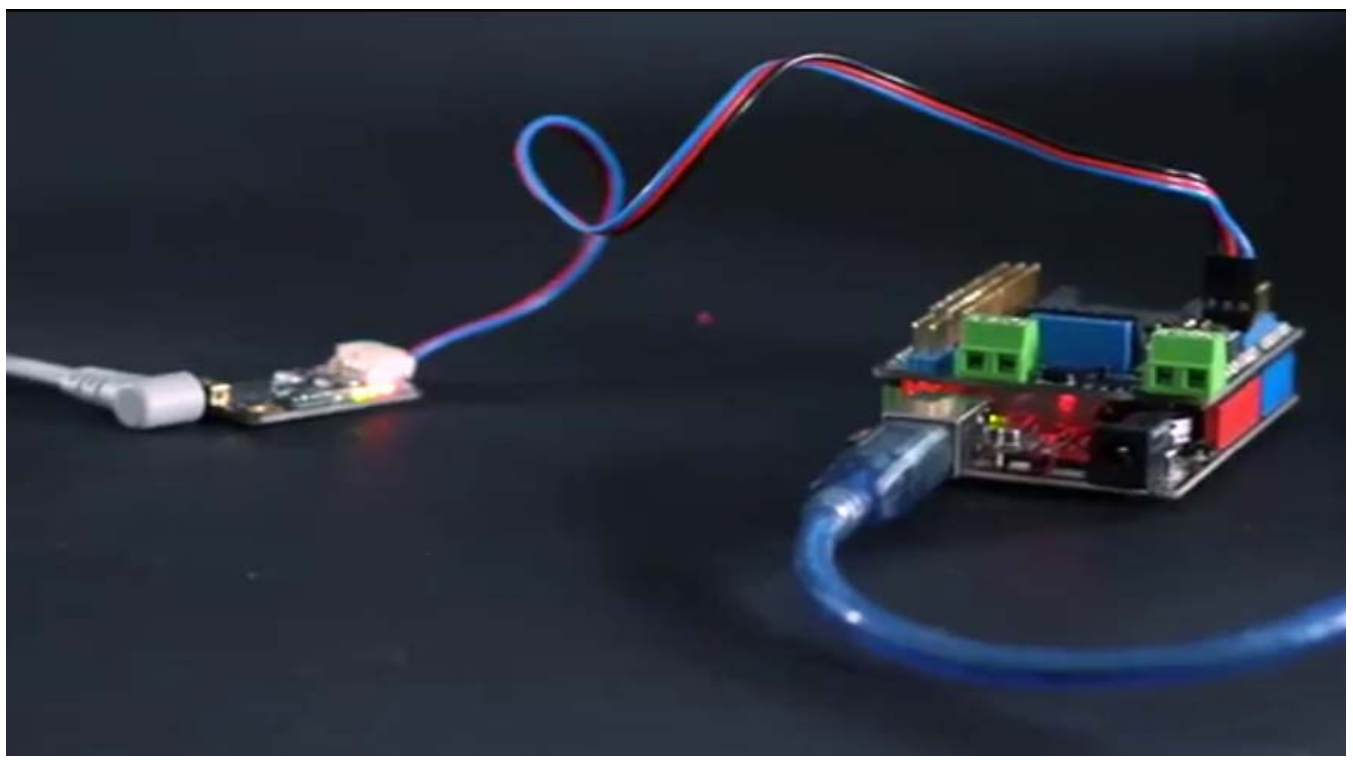

Figure 5: ESP 8266 Wi-Fi module connected with Arduino processor.

\section{Application layer}

The monitoring of collected information's by detecting layer, is done in the application layer, by retrieving the data's from cloud server. Here the smart phone android app Thing Speak is used for retrieve the data from open cloud server Thing Speak. The Thing Speak app is download and installed from google play store after that by using the API key get in the transport layer, open the created channel and check the data's. in this work, the live updating of information's happens so it is possible to monitor the live health status of subject such as body temperature, position of body and also the current location of subject and heart beat rate. 

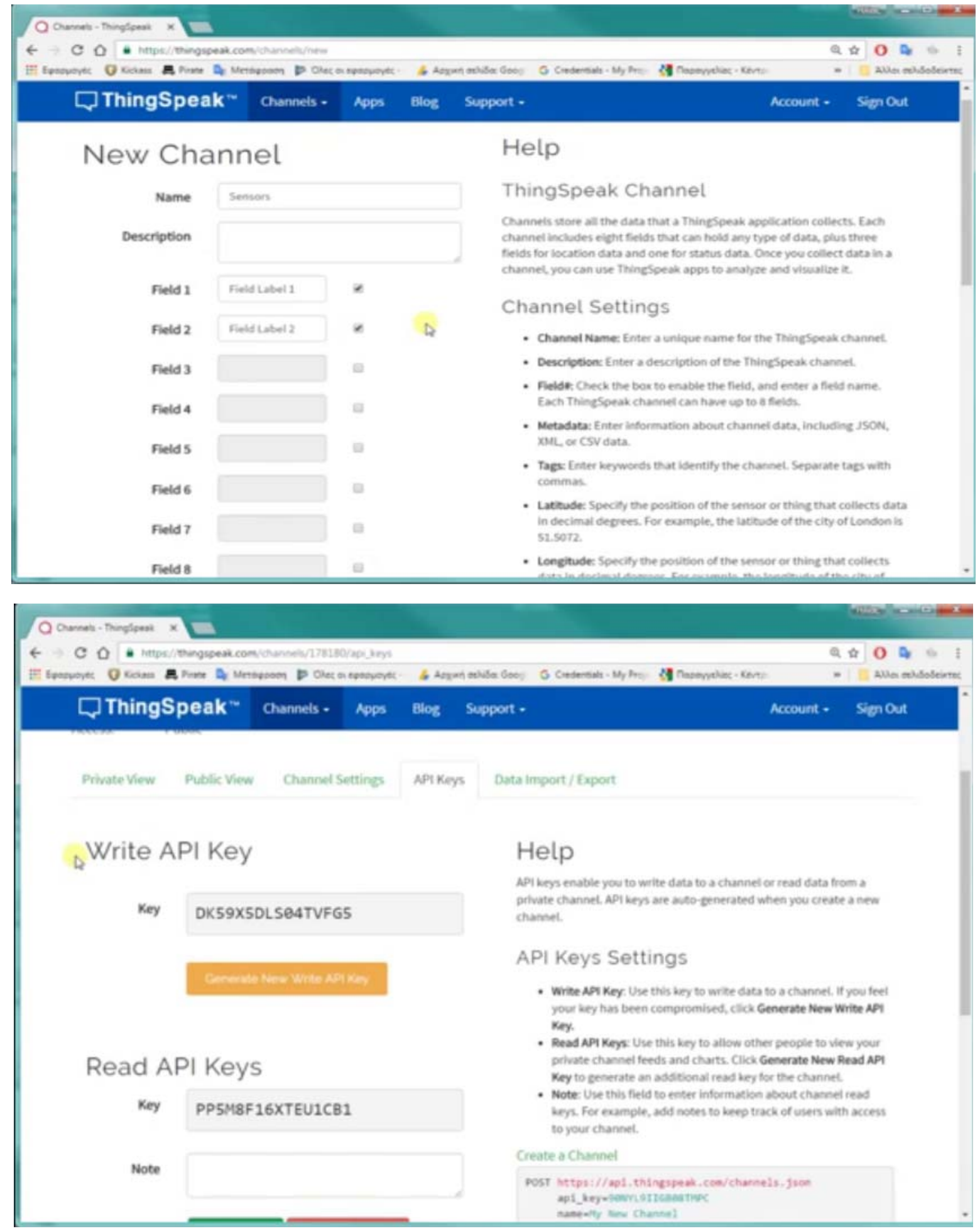

Figure 6 a: Creation of new channel \& Figure 6 b: Generation of API key

\section{CONCLUSION}

In this paper, an advanced methodology using IOT and WBASN is introduced for analyze the real time health status of human. The vital health parameters of subject are collected by using three sensors such temperature sensor, accelerometer and ECG/EMG sensors, the collected data are preprocessed by an Intel Galileo processor and send to an open cloud server Thing Speak by using a wireless communication Wi-Fi, so that the real time health status of subject is upload to the cloud in a live manner, from the cloud the health care professions can analyze the collected information's by using their Smartphone android app Thing Speak by login it. Furthermore, this work can be extended by associating more sensors, for collect more vital physiological parameters of subject. 


\section{REFERENCES}

[1] Subhas Chandra Mukhopadhyay," Wearable Sensors for Human ActivityMonitoring: A Review", IEEE Sensors Journal, Vol. 15, No. 3, pp 1321-1330, March 2015.

[2] Bourouis, A., Feham, M., and Bouchachia, A.(2011), “ Ubiquitous Mobile Health Monitoring System for Elderly (UMHMSE)”, International Journal of Computer Science and Information Technology,Vol.2, No. 3, June, pp. 74-82

[3] G. Fortino and P. Trunfio, "Internet of things based on smart objects: technology, middleware and applications," Cham, Switzerland: Springer, 2014.

[4] P. Gope and T. Hwang, "Bsn-care: a secure iot-based modern healthcare system using body sensor network," IEEE Sensors Journal, vol. 16, no. 5, pp. 1368-1376, 2016.

[5] R. Gravina, P. Alinia, H. Ghasemzadeh, and G. Fortino, "Multi-sensor fusion in body sensor networks: State-of-the-art and research challenges,” Information Fusion, vol. 35, pp. 68-80, 2017.

[6] M. M. Hassan, K. Lin, X. Yue, and J. Wan, "A multimedia healthcare data sharing approach through cloud-based body area network," Future Generation Computer Systems, vol. 66, pp. 48-58, 2017.

[7] Y.H.Kwak,W.Kim,K.B.Park,K.Kim,andS.Seo,“Flexibleheartbeat sensor for wearable device,” Biosensors and Bioelectronics, vol. 94, pp. 250-255, 2017.

[8] C. Seeger, K. Van Laerhoven, and A. Buchmann, "Myhealthassistant: An event-driven middleware for multiple medical applications on a smartphone-mediated body sensor network," IEEE journal of biomedical and health informatics, vol. 19, no. 2, pp. 752-760, 2015.

[9] Thingspeak, launched by ioBridge in 2016, available at: https://en.wikipedia.org/wiki/ThingSpeak/ and https://hingspeak.com/

[10] Andrea Zanella, Nicola Bui, Angelo Castellani, Lorenzo Vangelista, and Michele Zorzi, " Internet of Things for Smart Cities", IEEE Internet Of Things Journal, Vol. 1, No. 1, pp 22-32, February 2014.

[11] Y-C. Lee, C-C. Chou, W-C Fang, H-C. Huang, "Portable sleep monitoring and awaking system based on EEG, ECG, tri-axis accelerometers and LED array panel", IEEE International Conference on Consumer Electronics - Berlin (ICCE-Berlin), 6-8 Sept. 2011.

[12] S. M. Riazul Islam, Daehan Kwak, Md. Humaun Kabir, mahmud Hossain, And Kyung-sup Kwak, "The Internet Of Things For Health Care: A Comprehensive Survey”. IEEE Access, June 2015.

[13] Luca Catarinucci, Danilo de Donno, Luca Mainetti, Luca Palano, Luigi Patrono, Maria Laura Stefanizzi, and Luciano Tarricone. "An IoTAware Architecture for Smart Healthcare Systems”. IEEE JOURNAL, Dec 2015.

[14] Mohammad S. Jassas, Abdullah A. Qasem, Qusay H. Mahmoud “A Smart System Connecting e-Health Sensors and the Cloud”. IEEE conference May 2015.

[15] Dr. Salah S. Al-Majeed, Dr. Intisar S. Al-Mejibli, Prof. Jalal Karam. “Home Telehealth by Internet of Things (IoT)”.IEEE conference May 2015. 